\title{
DietCompLyf Study: baseline characteristics and dietary intake
}

\author{
S. F. Brennan ${ }^{1}$, M. M. Cantwell ${ }^{1}$, R. Swann ${ }^{2}$, C. Robertson ${ }^{2}$, A. Mulligan ${ }^{3}$, M. V. Dwek ${ }^{2}$ \\ and J. V. Woodside \\ ${ }^{1}$ Centre for Public Health, Queen's University Belfast, Institute of Clinical Sciences B, Belfast, BT12 6BJ, ${ }^{2}$ Against Breast \\ Cancer Research Unit, University of Westminster, London, UK and ${ }^{3} M R C$ CNC, Department of Public Health and Primary \\ Care, Institute of Public Health, University of Cambridge, UK
}

The DietCompLyf study is the first UK-based prospective cohort of breast cancer patients and was designed to investigate the association between phytoestrogen intake (from foods and supplements) and event-free survival. Information on pathology, treatment, lifestyle and demographic factors has been collected. The aim of this abstract is to examine dietary intakes in this cohort by common prognostic factors.

Breast cancer patients were recruited from 56 hospitals across the UK. Detailed information on pathology, treatment and medication was obtained from hospital staff and information on lifestyle was self-reported by patients at baseline (9-15 months post breast cancer diagnosis). Current dietary intake was estimated using the EPIC-Norfolk FFQ, within which participants indicated the frequency of consumption of 131 food and beverage items. Portions of fruit, vegetables and fish consumed per day were calculated from the frequencies obtained. The frequencies were also converted to nutrients using the Compositional Analysis from Frequency Estimates (CAFE) nutritional analysis program ${ }^{(1)}$. Mean daily intakes of twenty-eight nutrients were considered in the present analysis and percentage energy contributions from food groups were also calculated. Dietary intake was compared by tumour grade, lymph node status and breast cancer subtype, specifically triple negative (ER-/PR-/HER2-) breast cancer and all other breast cancer subtypes.

In total, data for 3157 breast cancer patients was available for analysis. Women who were diagnosed with grade 1 breast cancer reported a lower percentage energy intake from fat $(32.1 \%$; SD: $6.2 \%)$ compared with women diagnosed with grade 3 breast cancer (32.8\%; SD: $5.9 \%)(p=0.01)$. Those with a positive node status reported higher intakes of vitamin C $(149.1 \mathrm{mg} ; \mathrm{SD}: 79.1 \mathrm{mg} ; p=0.01)$, folate $(318.3 \mu \mathrm{g}$; SD: $114.7 \mu \mathrm{g} ; p=0.01)$, fibre $(18.4 \mathrm{~g}$; SD: $7.6 \mathrm{~g} ; p=0.01)$, vitamin A retinol equivalents $(1304.6 \mu \mathrm{g}$; sD: $868.8 \mu \mathrm{g}$; $p=0.01)$ and portions of vegetables (5.2; SD: $3.3 ; p=0.02)$ but lower calcium intake (849.9 mg; SD: 319.5 mg; $p=0.01)$ compared with women with no nodal involvement (vitamin C:142.2 mg, SD: $71.3 \mathrm{mg}$; folate: $307.6 \mu \mathrm{g}$, sD:101.8 $\mu \mathrm{g}$; fibre: $17.7 \mathrm{~g}$, sD: $6.9 \mathrm{~g}$; vitamin A retinol equivalents: $1227.5 \mu \mathrm{g}$, SD: $750.6 \mu \mathrm{g}$; vegetable portions: 4.9 , SD: 2.6 ; calcium $879.7 \mathrm{mg}$, SD: $309.5 \mathrm{mg}$, respectively). Women with triple negative breast cancer reported a higher intake of polyunsaturated fatty acids (13.5 g; SD: $6.2 \mathrm{~g} ; p=0.02)$ and vitamin E (12.6 mg; SD: $5.4 \mathrm{mg} ; p=0.01)$ compared with other breast cancer subtypes $(12.5 \mathrm{mg}$, SD $5.7 \mathrm{mg}$ and $12.6 \mathrm{mg}$, sD $5.4 \mathrm{mg}$, respectively).

Small and inconsistent differences in dietary intake were observed with common prognostic factors in DietCompLyf breast cancer patients. This is in contrast to some literature which suggests that prognostic factors, which may act as a motive for diet and lifestyle changes post-diagnosis ${ }^{(2)}$, influence dietary intake amongst early stage breast cancer survivors ${ }^{(3)}$.

1. Welch AA, Luben R, Khaw KT et al. (2005) J Hum Nutr Diet 18(2), 99-116.

2. Demark-Wahnefried W, Aziz NM, Rowland JH et al. (2005) J Clin Oncol 23(24), 5814-5830.

3. Salminen E, Heikkilä S, Poussa T et al. (2004) Prev Med 35(5), 529-535. 